\title{
Efeito da endogamia sobre características morfométricas em cavalos da raça Mangalarga Marchador
}

\author{
[Effect of inbreeding on morfhometric traits in Mangalarga Marchador horses]
}

\author{
R.W. Gonçalves ${ }^{1}$, M.D. Costa ${ }^{2 *}$, A.S.C. Rezende ${ }^{3}$, V.R. Rocha Júnior ${ }^{2}$, J.R.A. Leite ${ }^{4}$ \\ ${ }^{1}$ Aluno de pós-graduação - Unimontes - Janaúba, MG \\ ${ }^{2}$ Unimontes - Janaúba, MG \\ ${ }^{3}$ Escola de Veterinária - Universidade Federal de Minas Gerais - Belo Horizonte, MG \\ ${ }^{4}$ Aluno de graduação - Universidade Estadual de Montes Claros - Montes Claros, MG
}

\begin{abstract}
RESUMO
Estimou-se o coeficiente de endogamia e avaliou-se seu efeito sobre características morfométricas dos animais em um criatório no norte de Minas Gerais. As características estudadas foram alturas da cernelha e da garupa, comprimentos da cabeça, do pescoço, do dorso-lombo, da garupa e do corpo, larguras do peito e da garupa. $\mathrm{O}$ arquivo continha 2186 informações de parentesco de animais do arquivo zootécnico da fazenda, desde o início da formação da raça, 1951, até o ano de 2006. Calculou-se o coeficiente de endogamia (F) e avaliou-se seu efeito por meio de regressão linear simples sobre as medidas morfométricas. Do total de animais, $27,6 \%$ mostraram-se endogâmicos, sendo o F médio da população igual a 1,4\%. Considerando-se apenas os animais endogâmicos, a consanguinidade média atingiu 5,3\%, mínimo de 0,1 e máximo de $28,1 \%$. Não se verificaram efeitos negativos da endogamia sobre as características morfométricas, exceto para largura da garupa, em que se observou que para cada $10 \%$ de acréscimo de $\mathrm{F}$ houve aumento de $0,576 \mathrm{~cm}$ na largura da garupa. Possivelmente, devido ao baixo valor encontrado, a endogamia não influenciou as demais características avaliadas.
\end{abstract}

Palavras-chave: equinos, consanguinidade, depressão endogâmica, medidas lineares

\begin{abstract}
The coefficient of inbreeding was estimate and its effect on linear traits of the Mangalarga Marchador horses reared in a ranch in the North of Minas Gerais was evaluated. The characteristics studied were morphometric traits such as wither and hip height, length of head, neck, back-loin, hip and body, as well as chest and hip width. The archive had 2186 data on the genealogy of animals from the herd register of the Catuni Farm. The coefficient of inbreeding $(F)$ was calculated and its effect was evaluated by means of simple linear regression on the linear traits. Of all animals, $27.6 \%$ showed inbreeding, with an $F$ average of $1.4 \%$ in the population. Considering only the inbred animals, the average consanguinity reached 5.3\% minimum of 0.1 and maximum of $28.1 \%$. Negative effects of inbreeding on the morphometric traits were not seen, except for hip width, where for each $10 \%$ increase in $F$ there was increase of $0.576 \mathrm{~cm}$. Possibly due to the low value found, inbreeding did not influence the other evaluated characteristics.
\end{abstract}

Keywords: horse, consanguinity, inbreeding depression, linear measures

\section{INTRODUÇÃO}

A Mangalarga Marchador é a mais numerosa raça equina brasileira e encontra-se distribuída em todo o país. Tem sido utilizada, principalmente, para trabalhos no campo em fazendas de gado de corte, mas, nos últimos anos, tem sido crescente sua participação em variados tipos de esporte hípico, além de lazer em passeios equestres e no turismo ecológico (Costa et al., 2005).

Recebido em 27 de abril de 2011

Aceito em 31 de janeiro de 2012

*Autor para correspondência (corresponding author)

E-mail: dulcineia.costa@unimontes.br

Apoio: FAPEMIG-CVZ APQ-3335-5.04-07 
A linhagem Catuni representa importante pilar da raça Mangalarga Marchador, com animais que apresentam como característica marcante o andamento marchado. A seleção desses animais teve início com Casemiro Colares, na Fazenda Santa Helena, norte de Minas Gerais (Casiuch, 2005), que se tornou referência na região pela qualidade da tropa de cavalos de sela que criava. Foi usado o garanhão Pedra Estanho, adquirido da Fazenda Favacho, em meados de 1950, que permaneceu no criatório por 18 anos, sendo grande parte dos animais do plantel atual seus descendentes (Catuni, 2009).

Apesar da importância, na literatura consultada foram poucos os estudos encontrados sobre a endogamia em equídeos e sua influência sobre as características morfométricas, como altura na cernelha, comprimento do corpo, largura da cabeça dentre outras (Gandini et al.,1992; Bergmann et al., 1997; Zechner et al., 2001; Van Eldik et al., 2006), e, em animais da raça Mangalarga Marchador, as pesquisas são inexistentes. Dessa forma, este estudo teve como objetivo avaliar o coeficiente de endogamia e sua influência sobre as características morfométricas dos animais da raça Mangalarga Marchador criados na região.

\section{MATERIAL E MÉTODOS}

Foram avaliados dados de equinos da raça Mangalarga Marchador, pertencentes ao arquivo da Fazenda Catuni, do início da formação do rebanho, na década de 50, até o ano de 2006. O arquivo continha 2186 observações com as informações de identificação dos animais; data de nascimento do animal; identificação do pai; identificação da mãe; e as mensurações. A partir dos 36 meses e observadas as exigências do Regulamento de Registro Genealógico da Associação Brasileira dos Criadores do Cavalo Mangalarga Marchador (ABCCMM), os animais são submetidos a exame zootécnico, por técnico treinado e credenciado junto à ABCCMM. Nestas avaliações zootécnicas, os animais são mensurados com o auxílio de hipômetro metálico, empregando-se o sistema métrico e tendo como referência anatômica os pontos descritos por Barbosa (1993) e Zamborlini et al. (1996).
Foi calculado o coeficiente médio de endogamia (F) proposto por Wright (1922), com 2186 animais na matriz de parentesco. Para tanto, utilizou-se o programa MTDFNRM (Multiple-Trait Derivative-Free Numerator Relationship Matrix), componente do sistema MTDFREML (Multiple-Trait Derivative-Free Restricted Maximum Likelihood) (Boldman et al., 1995).

Os efeitos da endogamia sobre as características morfométricas altura na cernelha (AC), altura na garupa (AG), comprimento da cabeça (CC), comprimento do pescoço (CP), comprimento da garupa (CG), comprimento da espádua (CE), comprimento do corpo (CCorp), comprimento dorsolombar (CD), largura da cabeça (LC), largura do peito (LP), largura da garupa (LG), perímetro do tórax (PT) e perímetro de canela (PC) foram avaliados por meio de análise de variância, utilizando-se os procedimentos do software SAEG (Sistema...2000).

Para as características em que o efeito da endogamia foi significativo $(\mathrm{P}<0,05)$, efetuou-se análise de regressão. Para melhor compreensão do efeito da endogamia sobre as características estudadas, os coeficientes de endogamia (F) foram agrupados em sete classes, que variaram de zero a acima de $25 \%$ (Tab. 1).

Tabela 1. Classes dos coeficientes de endogamia de equinos da raça Mangalarga Marchador criados em um rebanho no norte de Minas Gerais

\begin{tabular}{lc}
\hline $\begin{array}{l}\text { Classe de } \\
\text { endogamia }\end{array}$ & $\begin{array}{c}\text { Coeficiente de } \\
\text { endogamia }(\%)\end{array}$ \\
\hline 1 & 0 \\
2 & 0 a 5 \\
3 & 5 a 10 \\
4 & 10 a 15 \\
5 & 15 a 20 \\
6 & 20 a 25 \\
7 & $>25$ \\
\hline
\end{tabular}

Ainda, com o objetivo de verificar a flutuação do coeficiente de endogamia desde a formação do rebanho até os dias atuais, os dados foram distribuídos por períodos de 10 anos correspondentes, aproximadamente, ao intervalo médio de geração dos animais da raça Mangalarga Marchador (Costa et al., 2005). 


\section{RESULTADOS E DISCUSSÃO}

O coeficiente médio de endogamia calculado para o rebanho foi de $1,4 \%$. Quando se avaliaram apenas os animais endogâmicos, o $\mathrm{F}$ médio foi $5,3 \%$, mínimo de 0,1 e máximo de $28,1 \%$. Esses resultados são mais baixos do que os relatados por Procópio et al. (2003), que, na raça Campolina, encontraram $\mathrm{F}$ de $6,0 \%$, e acima dos relatados por Costa et al. (2005), que encontraram, na população de Mangalarga Marchador, F de 5,0\%. Este baixo valor pode estar associado à utilização, na reprodução, de animais geneticamente diferentes, refletindo pouca ocorrência de acasalamentos entre parentes.
Os valores de $\mathrm{F}$ mantiveram-se acima de $10 \%$, para aproximadamente $4,4 \%$ da população analisada (Tab. 2). De acordo com Bergmann et al. (1997), F acima de $10 \%$ pode provocar depressão endogâmica, isto é, diminuição das características produtivas e, principalmente, das características adaptativas.

Entre o período de 1951 e 1960, correspondente ao período de início da formação do rebanho, o coeficiente de endogamia foi zero, e entre o período de 1961 e 1970, os valores foram mais elevados (Fig. 1).

Tabela 2. Número $(\mathrm{N})$ e percentual $(\%)$ de indivíduos, coeficiente médio de endogamia $(\mathrm{F})$, respectivos desvios-padrão (DP) dos animais da raça Mangalarga Marchador em um rebanho no norte de Minas Gerais, de acordo com a classe do coeficiente de endogamia

\begin{tabular}{lcccc}
\hline Classe de F & N & $\%$ & F & DP \\
\hline 0 & 1583 & 72,41 & 0 & 0 \\
0 a 5 & 382 & 17,47 & 2,20 & 1,24 \\
5 a 10 & 125 & 5,71 & 6,60 & 0,88 \\
10 a 15 & 56 & 2,56 & 12,50 & 0,64 \\
15 a 20 & 24 & 1,09 & 16,96 & 1,40 \\
20 a 25 & 1 & 0,04 & 20,30 & - \\
$>25$ & 16 & 0,68 & 25,60 & 1,30 \\
\hline
\end{tabular}

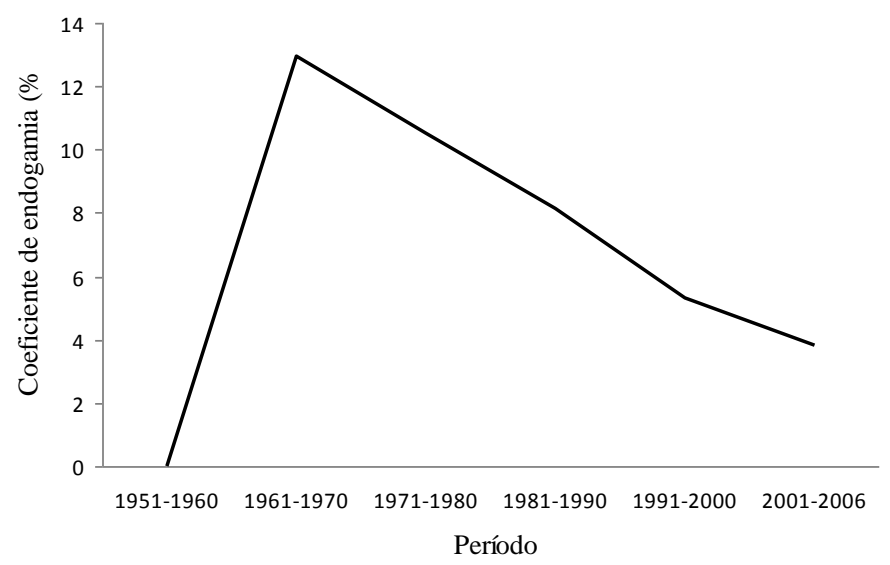

Figura 1. Comportamento do coeficiente médio de endogamia (F) de animais da raça Mangalarga Marchador criados em um rebanho no norte de Minas Gerais no período analisado.

Esse maior valor de $\mathrm{F}$, no segundo período, pode ser atribuído à intensa utilização dos reprodutores Pedra Estanho e Catuni El Toro. No período inicial, de 1951 a 1960 , foram utilizadas 63 éguas e cinco garanhões, que produziram 104 animais no período. Destes, 43 , ou $41,3 \%$, eram filhos de Pedra Estanho. No período seguinte, 1961 a 1970, foram utilizados nove garanhões, que deixaram 206 crias e, dessas, 63, ou 31,0\%, eram filhos de Pedra Estanho, e $64(31,0 \%)$ de Catuni El Toro, que é filho de Pedra Estanho. Portanto, só neste período, obtiveram-se, 
aproximadamente, $62,0 \%$ dos animais com parentesco próximo de Pedra Estanho, o que resultou em valor mais elevado de endogamia neste período.

Entre 1971 e 1980, verificou-se diminuição de F, o que pode ser considerado resultado de maior controle de acasalamentos. Nesse período, apesar de Catuni El Toro ter sido utilizado com intensidade elevada, outros garanhões de diferentes criatórios foram também utilizados como Patrimônio de Santa Lúcia, Soneto da Fazendinha, Frege de Passa tempo e Zircônio AJ, o que pode ter contribuído para o declínio da endogamia, já que não havia nenhum parentesco entre estes e o rebanho.

O efeito do reprodutor, pai do animal, foi importante fonte de variação $(\mathrm{P}<0,05)$ para todas as medidas lineares, exceto para altura na cernelha, altura na garupa e comprimento de cabeça, resultados semelhantes aos encontrados por Costa (1998) em animais da raça Pônei. Zechner et al. (2001) encontraram efeito significativo do reprodutor sobre perímetro torácico e largura da garupa, em cavalos Lipizzan. A constatação deste efeito evidencia a importância do componente genético aditivo nestas características.
O sexo teve efeito significativo $(\mathrm{P}<0,05)$ sobre todas as características avaliadas, exceto comprimento do dorso e largura da garupa (Tab. 3). Miserani et al (2002) não verificaram efeito significativo do sexo para comprimento do pescoço e da garupa e larguras da cabeça e da garupa para animais da raça Pantaneira. O comprimento do pescoço e as larguras da cabeça e da garupa estão associados com feminilidade, enquanto o comprimento da garupa favorece a impulsão dos membros posteriores, o que justifica esta diferença entre os machos e as fêmeas, já que os machos são mais utilizados no trabalho de campo.

As éguas estudadas apresentaram a altura na cernelha relativamente mais baixa do que a altura na garupa. De acordo com Ribeiro (1988), a altura na cernelha deve ser a mesma da garupa para uma boa proporção do animal. Estes valores refletem as determinações do padrão racial da raça Mangalarga Marchador, em que os machos, para serem registrados, devem ter as alturas da cernelha e da garupa iguais, e para registro das fêmeas, aceita-se altura na garupa de até dois centímetros superior à altura na cernelha (Associação..., 1991).

Tabela 3. Médias de medidas lineares de animais da raça Mangalarga Marchador criados em um criatório no norte de Minas Gerais, de acordo com o sexo

\begin{tabular}{lccc}
\hline Característica morfométrica & Pr>0,05 & Macho & Fêmea \\
\hline Altura na cernelha $(\mathrm{cm})$ & $0,0001^{*}$ & $150,04 \pm 2,90$ & $146,63 \pm 3,15$ \\
Altura na garupa $(\mathrm{cm})$ & $0,0001^{*}$ & $149,41 \pm 2,90$ & $147,11 \pm 3,12$ \\
Comprimento de cabeça $(\mathrm{cm})$ & $0,0093^{*}$ & $58,10 \pm 1,65$ & $57,09 \pm 2,73$ \\
Comprimento do corpo $(\mathrm{cm})$ & $0,0001^{*}$ & $152,0 \pm 4,01$ & $149,54 \pm 3,28$ \\
Comprimento do pescoço $(\mathrm{cm})$ & $0,0001^{*}$ & $62,15 \pm 2,76$ & $60,16 \pm 2,14$ \\
Comprimento da espádua $(\mathrm{cm})$ & $0,0001^{*}$ & $54,97 \pm 3,71$ & $52,39 \pm 2,41$ \\
Comprimento do dorso $(\mathrm{cm})$ & $0,2976^{\mathrm{ns}}$ & $54,06 \pm 6,11$ & $52,22 \pm 4,43$ \\
Largura da cabeça $(\mathrm{cm})$ & $0,0001^{*}$ & $20,51 \pm 0,81$ & $20,08 \pm 0,59$ \\
Comprimento da garupa $(\mathrm{cm})$ & $0,0218^{*}$ & $52,51 \pm 2,30$ & $51,88 \pm 2,19$ \\
Largura da garupa $(\mathrm{cm})$ & $0,5604^{\mathrm{ns}}$ & $50,37 \pm 1,62$ & $50,02 \pm 1,88$ \\
Perímetro do tórax $(\mathrm{cm})$ & $0,0001^{*}$ & $174,48 \pm 5,99$ & $170,0 \pm 6,09$ \\
Perímetro da canela $(\mathrm{cm})$ & $0,0001^{*}$ & $18,17 \pm 0,71$ & $17,48 \pm 0,59$ \\
\hline
\end{tabular}

ns=não significativo $(\mathrm{P}>0,05) ; *=$ significativo $(\mathrm{P}<0,05)$.

As médias de comprimento da cabeça foram de $58,10 \mathrm{~cm}$ nos machos e de $57,09 \mathrm{~cm}$ nas fêmeas. Estes resultados estão de acordo com Barbosa (1993) e Zamborlini et al. (1996), que encontraram valores de 58 e $57 \mathrm{~cm}$, respectivamente, para machos e fêmeas da raça Mangalarga Marchador. Também a largura da cabeça foi levemente maior para os machos. Zamborlini et al. (1996) verificaram o mesmo valor de $20 \mathrm{~cm}$ para machos e fêmeas adultos, e Barbosa (1993) constatou valores de 20,7 e 20,3cm para machos e fêmeas da mesma raça, respectivamente. Essa medida juntamente com o comprimento da cabeça, constitui variável 
importante, pois é indicativo da expressão racial dos animais, além de, na fêmea, demonstrar feminilidade. A cabeça é desproporcional quando a largura é superior à terça parte do comprimento, o que pode descaracterizar o animal racialmente, mesmo que possua ótima conformação das outras regiões do corpo (Berbari Neto, 2005).

Os machos apresentaram comprimento do pescoço em torno de $2 \mathrm{~cm}$ maior que as fêmeas. Esses valores foram próximos aos encontrados por Zamborlini et al. (1996), de 63 e $61 \mathrm{~cm}$, e por Barbosa (1993), de 62,7 e 61,4cm, e inferiores aos observados por Cabral et al. (2004), de 66,0 e $63,3 \mathrm{~cm}$, respectivamente, para machos e fêmeas adultos da mesma raça. Esta diferença, provavelmente, pode estar relacionada com a amostragem.

O comprimento do corpo dos machos foi maior que o das fêmeas, em torno de $3,0 \mathrm{~cm}$. Esses valores pouco diferiram dos observados por Zamborlini et al. (1996), de 152 e $148 \mathrm{~cm}$, mas foram maiores que os de Barbosa (1993), $150 \mathrm{e}$ $147 \mathrm{~cm}$. Como os machos apresentaram medidas de alturas na cernelha e na garupa mais elevadas do que as fêmeas, esperava-se que o comprimento do corpo também fosse mais elevado.

Os machos também apresentaram maior comprimento das espáduas que as fêmeas. Zamborlini et al. (1996) observaram valores médios de 54 e $51 \mathrm{~cm}$ para machos e fêmeas, respectivamente, e Lage et al. (2009), de 59 e $62 \mathrm{~cm}$, para o comprimento das espáduas dos machos e das fêmeas adultos da raça Mangalarga Marchador. Segundo os autores, esses resultados podem ser consequência do regime alimentar e de treinamento diferenciado impostos aos animais dos dois sexos.

O comprimento da garupa apresentou valores médios de $52,58 \mathrm{~cm}$ nos machos e de $51,88 \mathrm{~cm}$ nas fêmeas. Resultado similar foi encontrado por Barbosa (1993), 52,6 e 52,2cm. Outros autores (Zamborlini et al., 1996; Cabral et al., 2004), apesar de terem observado valores maiores para os machos, 54 e $54,6 \mathrm{~cm}$, respectivamente, verificaram que o mesmo fato não ocorreu nas fêmeas, que tiveram essas medidas reduzidas, 51 e 47,0cm. Em estudos recentes, Lage et al.(2009) verificaram que machos e fêmeas da mesma raça tiveram o mesmo comprimento de garupa, $54 \mathrm{~cm}$. A garupa é a região que, além de centro de impulsão, é o agente de transmissão da força da sua musculatura e daquela proveniente dos membros posteriores (Fontes, 1954), e sua superioridade pode estar associada à maior intensidade de treinamento físico nos machos.

O perímetro torácico define a capacidade cardiorrespiratória do animal, e o cavalo, por ser um atleta em potencial, deverá apresentar mensurações compatíveis com esta capacidade (Berbari Neto, 2005). Os machos tiveram médias maiores do que as das fêmeas, 174,60 e $170,54 \mathrm{~cm}$, respectivamente, atribuídas possivelmente à maior intensidade de treinamento físico. Esses valores foram inferiores aos observados em machos e fêmeas adultos, por Cabral et al. (2004), de 180,8 e $175,7 \mathrm{~cm}$, e por Lage et al. (2009), de 181,0 e $176,0 \mathrm{~cm}$, respectivamente.

O perímetro da canela está relacionado com a qualidade óssea do esqueleto e com a funcionalidade da região anatômica, já que determina, juntamente com o perímetro torácico, a capacidade de carga do animal (Berbari Neto, 2005). Os valores médios observados para o perímetro da canela estão de acordo com Zamborlini et al. (1996), que observaram que ambos, machos e fêmeas da raça Mangalarga Marchador, apresentaram média de $18 \mathrm{~cm}$; enquanto Barbosa (1993) encontrou valores médios de 18,7 e $17,9 \mathrm{~cm}$ para machos e fêmeas adultos da mesma raça, respectivamente.

$\mathrm{Na}$ grande maioria das características morfométricas, os machos foram maiores que as fêmeas. Isto, provavelmente, deve-se ao dimorfismo sexual e à maior pressão seletiva por ocasião do registro definitivo dos machos, futuros garanhões. O padrão racial da ABCCMM permite altura na cernelha variando de 147 a $157 \mathrm{~cm}$, com altura ideal de $152 \mathrm{~cm}$ para machos. Para fêmeas, seria de 140 a $152 \mathrm{~cm}$, sendo ideal a altura de $146 \mathrm{~cm}$. Arnason (1984) observou que o sexo influenciou todas as medidas corporais de animais da raça Icelandic Toelter na Islândia.

O mês de nascimento teve efeito significativo sobre altura na garupa, comprimento de cabeça, comprimento do corpo, comprimento da espádua, largura da cabeça, largura da garupa, 
perímetro do tórax e perímetro da canela (Tab. 4).

Houve variação mensal nas características morfométricas dos animais ao longo dos anos. Os animais que nasceram nos meses de julho e agosto apresentaram maiores médias para todas as características avaliadas. Animais nascidos no final do inverno (julho e agosto) são oriundos de éguas que entraram em reprodução e conceberam no início da estação de monta (setembro e outubro) e, por conseguinte, desmamaram os potros em dezembro e janeiro. Sabe-se, também, que os equinos apresentam taxa inicial de crescimento elevada e com seis meses de idade já atingiram $80 \%$ da altura final, desde que o manejo nutricional seja adequado. Assim, animais que desmamaram em condições nutricionais favoráveis provavelmente apresentaram melhores ganhos no desenvolvimento das características morfométricas do que aqueles que passaram por restrição alimentar por ocasião da desmama.

Tabela 4. Médias (cm) das características morfométricas de animais da raça Mangalarga Marchador em um criatório no norte de Minas Gerais, de acordo com o mês de nascimento

\begin{tabular}{|c|c|c|c|c|c|c|c|c|c|c|c|}
\hline \multirow{2}{*}{$\begin{array}{l}\text { Característica } \\
\text { morfométrica }\end{array}$} & \multirow{2}{*}{$\operatorname{Pr}>F$} & \multicolumn{10}{|c|}{ Mês } \\
\hline & & 1 & 2 & 3 & 4 & 7 & 8 & 9 & 10 & 11 & 12 \\
\hline $\mathrm{AC}$ & NS & 147,8 & 147,7 & 148,0 & 145,8 & 154,5 & 150,2 & 148,6 & 147,4 & 148,0 & 147,9 \\
\hline AG & $*$ & 147,4 & 147,8 & 148,2 & 145,3 & 154,5 & 149,3 & 148,7 & 147,5 & 148,3 & 148,0 \\
\hline CCAB & $*$ & 57,5 & 57,2 & 57,2 & 57,0 & 60,0 & 57,5 & 58,1 & 57,0 & 57,6 & 57,4 \\
\hline CCOR & $*$ & 149,9 & 150,5 & 150,0 & 147,8 & 156,0 & 152,7 & 151,6 & 150,3 & 151,2 & 150,3 \\
\hline CPES & NS & 60,8 & 60,0 & 60,1 & 59,8 & 62,5 & 61,1 & 62,6 & 60,9 & 60,9 & 60,6 \\
\hline CESP & NS & 52,6 & 51,9 & 52,1 & 51,3 & 57,0 & 55,1 & 54,5 & 53,6 & 53,4 & 52,6 \\
\hline CDOR & NS & 51,1 & 51,1 & 51,1 & 49,5 & 60,5 & 59,0 & 53,3 & 53,3 & 52,8 & 52,0 \\
\hline LCAB & NS & 20,3 & 20,2 & 20,4 & 19,3 & 21,0 & 20,5 & 20,3 & 20,2 & 20,2 & 20,2 \\
\hline CGAR & NS & 52,3 & 52,9 & 51,6 & 49,8 & 52,5 & 54,1 & 51,9 & 51,6 & 52,2 & 52,5 \\
\hline LGAR & NS & 50,1 & 50,5 & 49,1 & 48,8 & 51,0 & 51,8 & 50,7 & 49,7 & 50,4 & 50,2 \\
\hline PTOX & $*$ & 172,0 & 173,7 & 169,6 & 172,8 & 179,5 & 176,0 & 173,6 & 170,7 & 171,4 & 172,7 \\
\hline PCAN & NS & 17,8 & 17,9 & 17,6 & 17,5 & 18,0 & 18,0 & 17,9 & 17,7 & 17,7 & 17,8 \\
\hline
\end{tabular}

Observou-se efeito significativo $(\mathrm{P}<0,005)$ do ano de nascimento sobre todas as características avaliadas, exceto para altura na cernelha, comprimento da cabeça e comprimento da garupa. Esse resultado pode estar relacionado ao manejo em geral do rebanho e ao efeito do ambiente associados às variações nas mensurações feitas por diferentes técnicos de registro.

O efeito da endogamia dos animais da raça Mangalarga Marchador foi significativo apenas para largura da garupa (Tab. 5). Verificou-se que, para cada $10 \%$ de aumento de $\mathrm{F}$, houve aumento de $0,576 \mathrm{~cm}$ da largura da garupa
(Fig. 3). No entanto, esse resultado deve ser considerado com cautela, já que o coeficiente de regressão $\left(R^{2}=0,0223\right)$ foi baixo.

Quando se consideraram apenas os animais com endogamia acima de 5,3\%, seu efeito foi significativo $(\mathrm{P}<0,05)$ sobre os comprimentos da cabeça, do corpo, da espádua e da garupa. Para cada $1 \%$ de aumento em $\mathrm{F}$, houve aumento de $0,038 \mathrm{~cm} ; \quad 0,169 \mathrm{~cm} ; 0,156 \mathrm{~cm} ; 0,012 \mathrm{~cm}$ nos comprimentos da cabeça, do corpo, da espádua, da garupa, respectivamente, apesar de o coeficiente de regressão não ter sido significativo. 
Efeito da endogamia...

Tabela 5. Médias e significância estatística do efeito do coeficiente de endogamia sobre as características morfométricas de animais da raça Mangalarga Marchador criados em um criatório no norte de Minas Gerais

\begin{tabular}{lcc}
\hline Característica morfométrica & Média $(\mathrm{cm})$ & $\operatorname{Pr}>\mathrm{F}$ \\
\hline Altura na cernelha & 147,89 & $0,6229 \mathrm{~ns}$ \\
Altura na garupa & 147,95 & $0,4091 \mathrm{~ns}$ \\
Comprimento de cabeça & 57,43 & $0,9276 \mathrm{~ns}$ \\
Comprimento do corpo & 150,62 & $0,0817 \mathrm{~ns}$ \\
Comprimento do pescoço & 60,83 & $0,5635 \mathrm{~ns}$ \\
Comprimento da garupa & 52,13 & $0,8944 \mathrm{~ns}$ \\
Comprimento da espádua & 53,22 & $0,7311 \mathrm{~ns}$ \\
Comprimento do dorso & 52,64 & $0,4900 \mathrm{~ns}$ \\
Largura da cabeça & 20,23 & $0,2300 \mathrm{~ns}$ \\
Largura da garupa & 50,16 & $0,0414^{*}$ \\
Perímetro do tórax & 172,06 & $0,3240 \mathrm{~ns}$ \\
Perímetro da canela & 17,74 & $0,5425 \mathrm{~ns}$ \\
\hline
\end{tabular}

ns=não significativo $(\mathrm{p}>0,05) ;{ }^{*}=$ significativo $(\mathrm{p}<0,05)$.

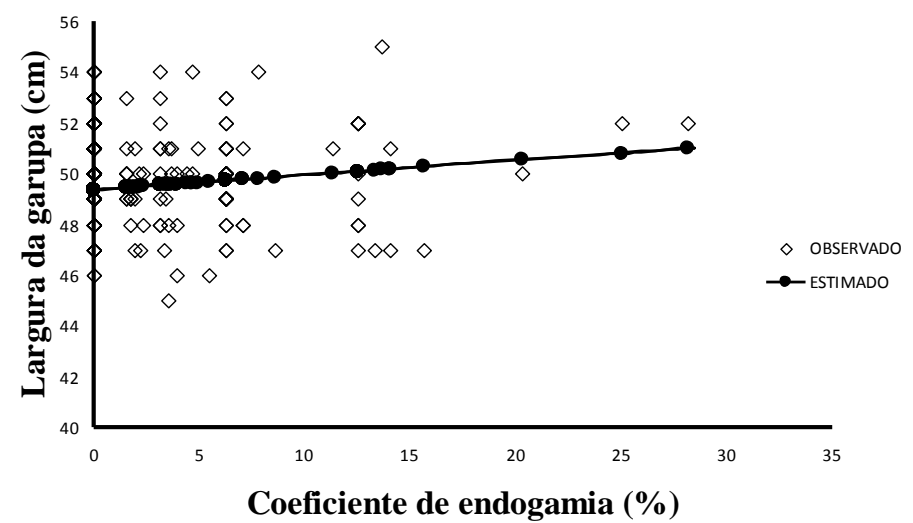

Figura 3. Representação gráfica do efeito do coeficiente de endogamia sobre a largura da garupa de animais da raça Mangalarga Marchador em um criatório no norte de Minas Gerais.

Estudos abordando o efeito da endogamia sobre características morfométricas em equídeos são escassos. Na raça Haflinger Italiana, Gandini et al. (1992) verificaram redução significativa de $1,1 \mathrm{~cm}$ e $2,9 \mathrm{~cm}$ para altura na cernelha e perímetro torácico, respectivamente, com aumento de $10 \%$ na endogamia. Bergmann et al. (1997), em pôneis da raça brasileira, verificaram que, independentemente da significância estatística, todos os coeficientes de regressão das características morfométricas sobre a endogamia (F) apresentaram sinal negativo. Nesta raça, um dos objetivos da seleção é reduzir o tamanho dos animais que podem alcançar preços mais elevados no mercado. Portanto, a endogamia foi favorável ao objetivo dos criadores.

A depressão endogâmica é mais provável de ocorrer em características relacionadas à reprodução e produção (Hansson e Westerberg, 2002), enquanto características morfométricas mostraram pouca ou nenhuma mudança (Van Eldik et al., 2006).

\section{CONCLUSÕES}

Baixos valores de endogamia não comprometem as características morfométricas. Se os acasalamentos são direcionados, a endogamia será mantida em valores ínfimos, evitando seu efeito desfavorável.

\section{AGRADECIMENTOS}

À Fundação de Apoio à Pesquisa e Ensino do Estado de Minas Gerais (FAPEMIG) e à CAPES, pelo apoio financeiro, e à Fazenda Catuni, pela cessão dos dados. 


\section{Gonçalves et al.}

\section{REFERÊNCIAS}

ÁRNASON, T. Genetic studies on conformation and performance of Icelandic Toelter horses. 1. Estimation of non-genetic effects and genetic parameters. Acta Agric. Scand. v.34, p.409-427, 1984.

ASSOCIAÇÃO Brasileira dos Criadores do Cavalo Mangalarga Marchador (ABCCMM). A História do Cavalo Mangalarga Marchador. Belo Horizonte: Associação Brasileira dos Criadores do Cavalo Mangalarga Marchador, 1991. p.89.

BARBOSA, C.G. Estudo morfométrico na raça Mangalarga Marchador: uma abordagem multivariada. 1993. 76f. Dissertação (Mestrado em Zootecnia) - Escola de Veterinária, Universidade Federal de Minas Gerais, Belo Horizonte.

BERBARI NETO, F. Evolução de medidas lineares e avaliação de índices morfométricos em garanhões da raça Campolina. 2005. 90f. Dissertação (Mestrado em Zootecnia) - Universidade Estadual do Norte Fluminense, Campos dos Goytacazes, RJ.

BERGMANN, J.A.G.; COSTA, M.D.; MOURÃO, G.B. et al. Formação e Estrutura Genética da Raça Pônei Brasileira. Arq. Bras. Med. Vet. Zootec., v.49, p.251-259, 1997.

BOLDMAN, K.G.; KRIESE, L.A.; VAN VLECK, L.D. et al. A manual for use of MTDFREML: A set of programs to obtainestimates of variances and covariances(DRAFT). Lincoln: Department of Agriculture/Agricultural Research Service, 1995. 120 p.

CABRAL, G.C.; ALMEIDA, F.Q.; QUIRINO, C.R. et al. Avaliação morfométrica de equinos da raça Mangalarga Marchador: medidas lineares. Rev. Bras. Zootec., v.33, p.989-1000, 2004.

CASIUCH, R.L. O romance da raça: História do cavalo Mangalarga Marchador. São Paulo: Empresa das Artes, p.254, 1997.

CATUNI. Disponível em:

<http://www.Catuni.com.br> Acessado em: 10 nov. 2009.

COSTA, M.D.; BERGMANN, J.A.G.; PEREIRA, C.S. et al. Avaliação dos fatores genéticos e de ambiente que interferem nas medidas lineares dos pôneis da raça Brasileira. Rev. Bras. Zootec., v.27, p.491-497, 1998.
COSTA, M.D.; BERGMANN, J.A.G.; RESENDE, A.S.C. et al. Análise temporal da endogamia e do tamanho efetivo da população de equinos da raça Mangalarga Marchador. Arq. Bras. Med.Vet. Zootec., v.57, p.112-119, 2005.

GANDINI, G.C.; BAGNATO, A.; MIGLIOR, F. Inbreeding in the Italian Haflinger horse. J. Hered., v.109, p.433-443, 1992.

HANSSON, B.; WESTERBERG, L. Invited review on the correlation between heterozygosity and fitness in natural populations. J. Hered., v.11, p.2467-2474, 2002.

LAGE, M.C.G.R.; BERGMANN, J.A.G.; PROCÓPIO, A.M. et al. Associação entre medidas lineares e angulares de equinos da raça Mangalarga Marchador. Arq. Bras. Med. Vet. Zootec., v.61, p.968979, 2009.

MISERANI, M.G.; MCMANUS, C., SANTOS, S. A. et al. Avaliação dos Fatores que Influem nas Medidas Lineares do Cavalo Pantaneiro. Rev. Bras. Zootec., v.31, p.335-341, 2002.

PROCÓPIO, A.M.; BERGMANN, J.A.G.; COSTA, M.D. Formação e demografia da raça Campolina. Arq. Bras. Med. Vet. Zootec., v.55, p.361-365, 2003.

RIBEIRO, D.B. O cavalo de raças, qualidade $e$ defeitos. Rio de Janeiro: Editora Globo Rural, 1988. 290p.

SISTEMA de análises estatísticas - SAEG. Versão 8.0. Viçosa: UFV.2000.

VAN ELDIK, P., VAN DER WAAIJ, E.H., DUCRO, B. et al. Possible negative effects of inbreeding on sêmen quality in Shetland pony stallions. Theriogenology, v.65, p.1159-1170, 2006.

ZAMBORLINI, L.C.; BERGMANN, J.A.G.; PEREIRA, C.S. et al. Estudo genético quantitativo de medidas lineares da raça Mangalarga Marchador - 1 . Estimativas de fatores de ambiente e parâmetros genéticos. Rev. Bras. Cienc. Vet., v.3, p.33-37, 1996.

ZECHNER, F. Z., SÖLKNER, I. B., HABE, E.M. et al. Morphological description of the Lipizzan horse population. Livest. Prod. Sci., v.69, p.163-177, 2001. 